\title{
Long non-coding RNA growth arrest-specific transcript 5 in tumor biology (Review)
}

\author{
$\mathrm{XIN} \mathrm{YU}^{1,2^{*}}$ and ZHENG LI ${ }^{1 *}$ \\ ${ }^{1}$ Department of Orthopaedic Surgery, Peking Union Medical College Hospital, Peking Union Medical College, Beijing 100730; \\ ${ }^{2}$ State Key Laboratory of Cardiovascular Disease, Fuwai Hospital, National Center for Cardiovascular Diseases, \\ Chinese Academy of Medical Sciences and Peking Union Medical College, Xicheng, Beijing 100037, P.R. China
}

Received August 5, 2014; Accepted May 14, 2015

DOI: $10.3892 / \mathrm{ol} .2015 .3553$

\begin{abstract}
The recognition of the biological relevance of long non-coding RNA (lncRNA) molecules has only recently been recognized as one of the most significant advances in contemporary molecular biology. A growing body of evidence indicates that lncR NAs act not only as the intermediary between DNA and protein but also as significant protagonists of cellular functions. The dysregulation of lncRNAs has increasingly been linked to numerous human diseases, particularly cancers. Recent studies have demonstrated that the lncRNA growth arrest-specific transcript 5 (GAS5) was pervasively downexpressed in most human cancers compared with non-cancerous adjacent tissues including gastric, breast, lung and prostate cancer. In addition, patients with decreased GAS5 expression have a significantly poorer prognosis than those with higher expression. Furthermore, GAS5 is involved in the control of cell apoptosis, proliferation, metastasis, angiogenesis, DNA repair and tumor cell metabolism. This review provides an overview of the current knowledge concerning the role of GAS5 in tumor expression and biology function.
\end{abstract}

\section{Contents}

1. Introduction

2. Structural characterization and biological functions of GAS5

3. GAS5 status in cancers

4. GAS5 tumor-suppressive functions

5. Molecular mechanism and action of GAS5

6. Implications in cancer management

7. Conclusions and future directions

Correspondence to: Dr Zheng Li, Department of Orthopaedic Surgery, Peking Union Medical College Hospital, Peking Union Medical College, 1 Shuaifuyuan, Beijing 100730, P.R. China

E-mail:kleeo@163.com

${ }^{*}$ Contributed equally

Keywords: growtharrest-specific transcript 5, long non-coding RNA, tumor suppressor, cancer

\section{Introduction}

A tumor suppressor gene is defined as a gene whose product normally inhibits tumor development $(1,2)$. Tumor suppressor gene mutation may predispose animals to cancer, which plays a critical role in tumorigenesis (3). Previously, protein-coding genes were considered to be the main compartment of tumor suppressors (4). However, more recent studies have revealed that non-coding RNAs, including microRNA and long non-coding RNA (lncRNA) may also play a significant role in tumor suppression $(5,6)$.

LncRNA is defined as a non-protein-coding RNA molecule longer than 200 nucleotides in length $(7,8)$. In past decades, these long non-coding transcripts were simply considered as transcriptional 'noise' or cloning artifacts (9). However, accumulating evidence has revealed that lncRNAs play key roles in a number of biological and pathological processes (10). In particular, the role of lncRNAs in human cancers has been thoroughly researched and multiple lines of study have demonstrated that lncRNAs act as oncogenes or tumor suppressors during tumorigenisis (11).

LncRNA growth arrest-specific transcript 5 (GAS5) was originally identified in 1988 (12-14). GAS5 was named due to its increased expression upon cell growth arrest (12). Since its identification, a number of studies have demonstrated that the expression of GAS5 is decreased in various malignancies, including breast, gastric, lung and prostate cancer (15-17). In this review, we discuss the current knowledge on GAS5 lncRNA, with a focus on its status in human tumors, mechanism in tumorigenesis, clinical implication, and regulation of gene expression in carcinogenesis.

\section{Structural characterization and biological functions of GAS5}

GAS5 was originally isolated in a study aimed at screening potential tumor suppressor genes that were highly expressed during growth arrest (14). GAS5, located on chromosome 1q25, is approximately $630 \mathrm{nt}$ in length (18). GAS5 is classified as a 5 '-terminal oligopyrimidine (5'TOP) gene since it possesses an oligopyrimidine tract characteristic of the 5'TOP class of genes (19). 5'TOP genes are defined as a class of genes that have a uncommon pyrimidine-rich 5'-terminal sequence (20). 
The mRNAs of 5'TOP genes accumulate in messenger ribonucleoprotein particles (mRNPs) during cell growth arrest. GAS5 comprises 12 exons, which are not conserved between human and mouse homologs. GAS5 introns encode 10 small nucleolar RNAs (snoRNAs) and 2 mature lncRNA isoforms: GAS5a and GAS5b. GAS5a is the main isoform at $77 \mathrm{nt}$ in length whereas GAS5b has only $45 \mathrm{nt}$, missing $32 \mathrm{nt}$. However, the putative open reading frame is small and poorly conserved $(13,21)$.

GAS5 is non-protein coding but its RNA is spliced, polyadenylated, and associated with ribosomes (22). The essential biological activity of GAS5 is mediated through the introns, which encode multiple snoRNAs (12). The functions of GAS5 are not well known as yet; however, it expresses multiple snoRNAs that regulate the biosynthesis of ribosomal RNA. GAS5 transcript is ubiquitously expressed in tissues, but it is unstable in proliferating cells (23). Its spliced RNA is low in growing cells, but is highly expressed during growth arrest (serum starvation, density arrest) or under inhibition of translation by cycloheximide, pactamycin or rapamycin (12). The increased the transcript level of GAS5 was considered to be caused by prolonged decay rates. GAS5 has also proven to be necessary for normal growth arrest in human T lymphocytes $(24,25)$. A number of studies have been conducted to elucidate the functions of GAS5 in humans. GAS5 is proven to be associated with the cell cycle and cell progression, and critical to normal growth arrest (26). GAS5 overexpression inhibits cell cycle progression while GAS5 inhibition decreases cell apoptosis and contributes to faster cell cycle progression $(27,28)$.

\section{GAS5 status in cancers}

Several lines of evidence have revealed aberrant expression of GAS5 in numerous human cancers (15-17). GAS expression is decreased in a number of cancer types, suggesting that GAS5 may function as a tumor suppressor. In addition, overexpression of GAS5 contributed to growth arrest in vitro in numerous cancer lines. In this section, we discuss the current findings of the expression of GAS5 in various human cancer types (Table I).

Breast cancer.GAS5 transcript levels were markedly decreased in cancerous tissues compared with adjacent non-cancerous tissue, using reverse transcription-quantitative polymerase chain reaction analysis (15). The reduced expression of GAS5 suggested its potential role in oncogenesis. Certain GAS5 transcripts (GAS52B, GAS53A and GAS5O1) were proven to induce growth arrest and apoptosis in human breast cancer cell lines (29). GAS5 IncRNA promoted the apoptosis of triple-negative and estrogen receptor-positive breast cancer cells. Reduced GAS5 expression attenuated the cellular responses to apoptotic stimuli in breast cancer. In addition, the extent of breast cancer cell death was directly proportional to cellular GAS5 levels, indicating a correlation between GAS5 expression and prognosis of breast cancer patients. There were a negative correlation between miR-21 and GAS5 expression in breast tumor specimens (30). It was considered that miR-21 and GAS5 might regulate each other in a similar way to the microRNA-mediated silencing of target mRNAs. Nevertheless, the target genes of IncRNAs vary between specific tissues and cell types. As a result, the target gene of GAS5 in different cancer types differs. Further studies are needed to identify the target genes of GAS5 in different cancers.

Lung cancer. GAS5 expression has also been associated with carcinogenesis and metastasis in non-small cell lung cancer (NSCLC) patients. In 72 specimens of NSCLC, GAS5 expression was reduced in cancer samples compared with adjacent unaffected normal lung tissues $(\mathrm{P}<0.05)$. The GAS5 expression level was correlated with tumor size and tumornode-metastasis stage $(\mathrm{P}<0.05)$. Furthermore, a functional role of GAS5 in NSCLC was also determined. GAS5 overexpression was demonstrated to promote cell growth arrest and induce apoptosis in NSCLC in vitro and in vivo (23).

Gastric cancer. A previous study compared HOX transcript antisense RNA (HOTAIR) expression levels in 89 gastric cancer tissues with expression levels in corresponding non-cancerous gastric tissue (16). GAS5 expression was significantly decreased in gastric cancer tissues. In addition, GAS5 expression levels correlated with larger tumor size and advanced pathological stage. Low GAS5 expression levels indicated poorer prognosis, with shorter disease-free survival and overall survival times, serving as an independent prognostic indicator for gastric cancer. Functional analysis demonstrated that GAS5 inhibited gastric cancer cell proliferation and induced apoptosis in vitro and in vivo. GAS5 overexpression decreased E2F1 and cyclin D1 expression while it increased P21 expression in gastric cells. In addition, GAS5 downexpression increased E2F1 and cyclin D1 expression while it decreased P21 expression levels. These results indicated that by regulating E2F1 and P21, GAS5 inhibited gastric cancer development through a mechanism of post-transcriptional regulation (16).

Pancreatic cancer. Similar to the findings of GAS5 in gastric cancer, studies of pancreatic cancer have demonstrated that GAS5 expression was markedly decreased in pancreatic cancer tissues compared with normal pancreatic ductal cells. GAS5 inhibited pancreatic cancer cell proliferation. Furthermore, cyclin-dependent kinase 6 (CDK6) expression was negatively regulated by GAS5 in vitro and in vivo. Knockdown of CDK6 partially abrogates GAS5-siRNA-induced cancer cell proliferation (28).

Renal cell carcinoma. The expression of GAS5 was significantly downregulated in renal cell carcinoma cells compared with normal control cells. Overexpression of GAS5 inhibited cell proliferation, migration and invasion potential and induced cell apoptosis in renal carcinoma cell lines $(31,32)$.

Bladder cancer. GAS5 expression was lower in both bladder cancer cell lines and human specimens. Overexpression of GAS5 inhibited bladder cancer cell proliferation. GAS5 exerted its effect in bladder cancer partly by regulating CDK6, since knockdown of GAS5 increased CDK6 mRNA and protein levels in bladder cancer cells. GAS5 inhibition induces a significant decrease in the G0/G1 phase and a notable increase in the S phase (27).

Prostate cancer. GAS5 gene expression was significantly downregulated in prostate cell lines derived from metastases 
Table I. Growth arrest-specific transcript 5 expression in various human cancers.

\begin{tabular}{llcr}
\hline Cancer type & Expression & Method of analysis & Reference \\
\hline Breast cancer & Decreased & RT-qPCR & 15 \\
Lung cancer & Decreased & RT-qPCR & 23 \\
Gastric cancer & Decreased & RT-qPCR & 16 \\
Pancreatic cancer & Decreased & RT-qPCR & 28 \\
Renal cell carcinoma & Decreased & RT-qPCR \\
Bladder carcinomas & Decreased & RT-qPCR & 32 \\
Prostate cancer & Decreased & RT-qPCR \\
Malignant pleura mesothelioma & Decreased & RT-qPCR \\
B-cell lymphoma & Decreased & RT-qPCR \\
Leukemia & Decreased & RT-qPCR \\
\hline
\end{tabular}

RT-qPCR, reverse transcription-quantitative polymerase chain reaction.

(LNCaP and PC-3) compared with those derived from normal prostate tissue or primary prostate cancer $(17,33,34)$. In addition, the cellular GAS5 levels decreased as prostate cancer cells acquired castration resistance. GAS5 promoted the apoptosis of prostate cells. GAS5 overexpression promoted cancer cell death induced by UV-C irradiation and chemotherapeutic drugs, and downregulation of GAS5 expression attenuated it. LncRNA GAS5 binds to the corresponding domain on the androgen receptor and inhibits transcriptional stimulation (35). Considering that the androgen receptor plays a crucial role in the survival of prostate cancer cells (36), this is of potential significance, since downregulation of GAS5 levels may permit increased pro-survival signaling through the androgen receptor pathway.

Malignant pleura mesothelioma. GAS5 expression was significantly decreased in malignant pleura mesothelioma (MPM) cell lines compared with normal controls. GAS5 was largely expressed in quiescent cancerous cells. MPM cell growth arrest induced by drugs was accompanied with GAS5 accumulation in the nuclei. In addition, GAS5 expression was highly expressed in podoplanin-positive MPM. Silencing of GAS5 shortened the cell cycle length and increased the expression of glucocorticoid responsive genes, glucocorticoid inducible leucine-zipper and serum/glucocorticoid-regulated kinase-1 (22).

B-cell lymphoma. Nakamura et al reported a chromosomal translocation of $\mathrm{t}(1 ; 3)(\mathrm{q} 25 ; \mathrm{q} 27)$ in a case of diffuse large B-cell lymphoma (19). The chromosomal translocation of $t(1 ; 3)(q 25 ; q 27)$ led to the expression of GAS5-B-cell lymphoma 6 (BCL6) chimeric transcripts. In this case, the breakpoint in chromosome 1 was located within GAS5 and the breakpoint in chromosome 3 at $4 \mathrm{~kb}$ upstream of BCL6 exon 1a. Promoter substitution leads to the inappropriate expression of BCL6, which normally acts as a transcriptional repressor $(19,37)$. It was considered that the inappropriate expression of BCL6, which was a result of the changes in post-transcriptional regulation of the chimeric mRNA, was responsible for lymphomagenesis (19).
Leukemia. Etomidate is an essential tool in a variety of scenarios for intubation and procedural sedation. DNA microarray assay indicated that etomidate increased the expression of GAS5 in murine leukemia in vitro. Etomidate is cytotoxic due to its induction of apoptosis. However, the mechanism for etomidate-induced apoptosis remains unclear. GAS5 may play a role in the process (38). Table I summarizes the expression of GAS5 in various types of cancers.

\section{GAS5 tumor-suppressive functions}

The frequent downregulation of GAS5 in human cancers has provided new insight into our understanding of its functions. GAS5 suppresses tumors by regulating multiple cellular processes, including growth arrest, apoptosis, proliferation, metastasis and DNA damage repair. In this section, we discuss how GAS5 deregulations contribute to cancer development.

GAS5 regulates growth arrest and apoptosis. Apoptosis, also called programmed cell death, is critical in normal development, and in the initiation and progression of cancers (39). Mutated and deleted pro-apoptotic genes give rise to carcinogenesis and tumor growth. A number of in vitro studies have demonstrated that GAS5 is pro-apoptotic. In vitro and in vivo experiments revealed that GAS5 induced growth arrest and apoptosis in numerous cancers, including breast cancer, NSCLC, gastric cancer, renal cancer and prostate cancer $(15-17,23,31,40)$.

GAS5 regulates proliferation. GAS5 was demonstrated to inhibit tumor cell proliferation in gastric cancer, pancreatic cancer, renal carcinoma cell lines and bladder cancer cells $(16,27,28,31)$.

GAS5 regulates metastasis. In addition to promoting tumor cell apoptosis and inhibiting proliferation, GAS5 also inhibits tumorigenesis by inhibiting metastasis. Overexpression of GAS5 inhibited migration and invasion potential in renal carcinoma cell lines (31). GAS5 gene expression was markedly decreased in prostate cancer cells derived from metastases 
Cell Growth arrested

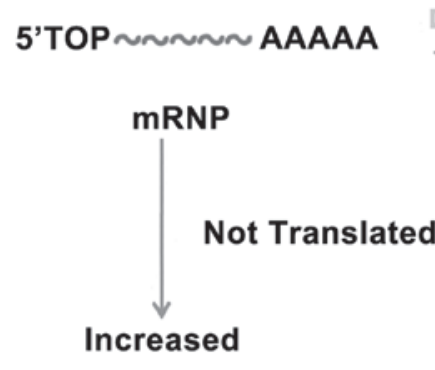

Cell Growing

5'TOP AAAAA

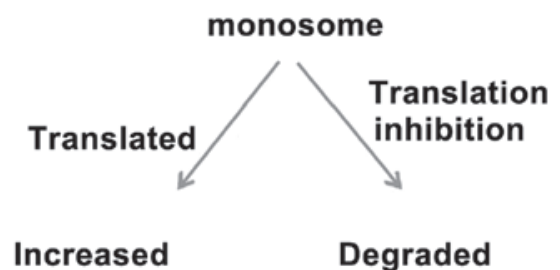

Figure 1. Cell growth arrest and growth arrest-specific transcript 5 (GAS5) expression. In growing cells, spliced GAS5 RNA is translated and consequently degraded. When translation is inhibited or cell growth is arrested, the levels of GAS5 increase. mRNP, messenger ribonucleoprotein particle.

when compared with those derived from normal prostate tissue or primary prostate cancer (34).

\section{Molecular mechanism and action of GAS5}

Although GAS5 has been reported as a tumor suppressor, the underlying molecular mechanism remains largely unknown. LncRNAs modulate the expression of various genes, playing a number of significant biological roles, including dosage compensation and genomic imprinting, histone-modification, gene activation, gene repression, lineage determination and cell proliferation $(5,41)$.

GAS5 controls gene transcription. Aberrant gene expression plays a critical role in the initiation and progression of cancer. Elucidation of the lncRNA-mediated transcriptional or post-transcriptional gene regulations has prompted investigations into the mechanisms of cancer.

The negative correlation between miR-21 and GAS5 expression in breast tumor suggested that they might regulate each other in a similar manner to the microRNA-mediated silencing of target mRNAs (30). In gastric cells, GAS5 overexpression decreased the expression of E2F1 and cyclin D1 and increased the expression of P21 (16). E2F1 was demonstrated to contribute to tumorigenesis by influencing cell proliferation (23). Cyclin D1 is also associated with the development of cancers through its involvement in cell cycle regulation (42). Furthermore, GAS5 snoRNA levels are strongly correlated with p53 expression in colorectal cancer (43). P53 plays a prominent role in cancer by deregulating apoptosis (44). In addition, p21 is downregulated or lost in several cancer types with its inhibitory control over the cell cycle $(45,46)$. GAS5 exerts its effect in bladder cancer partly by regulating CDK6, since knockdown of GAS5 increased CDK6 mRNA and protein levels $(27,28)$. CDK6 regulated the cell cycle, and dysregulation of CDK6 is associated with bladder cancer progression $(47,48)$. In addition, GAS5 expression was highly expressed in podoplanin-positive MPM (22). Podoplanin, a type I transmembrane sialomucin-like glycoprotein, is highly expressed in MPM and induces platelet aggregation (49). The GAS5-BCL6 chimeric transcripts resulting from the chromosomal translocation $\mathrm{t}(1 ; 3)(\mathrm{q} 25 ; \mathrm{q} 27)$ led to the inappropriate expression of BCL6 (19). It was considered to be responsible for the initiation and development of lymphoma.
GAS5 acts as molecular decoy sequestering-specific DNA-binding proteins. GAS5 could suppress glucocorticoid receptor (GR)-induced transcriptional activity of endogenous glucocorticoid-responsive genes by competing with DNA glucocorticoid receptor response element (GRE) for binding to the GR since it is structurally similar to GRE $(34,43)$. The GR regulates cell survival by binding the promoters of various glucocorticoid-responsive genes, including apoptosis-related genes (50).

Upstream regulation of GAS5 expression. As mentioned above, GAS5 expression increased upon cell growth arrest. GAS5 plays critical roles in normal growth arrest, as well as in the growth arrest of a number of cancers. However, how GAS5 expression increases upon cell growth arrest remains unclear. The classification of GAS5 as a 5'TOP gene provides a possible explanation as to why it functions as a growth arrest (20). During arrested cell growth, 5'TOP accumulates in mRNPs. In growing cells, spliced GAS5 RNA is associated with ribosomes, translated and rapidly degraded. When cell growth is arrested and translation is inhibited, the levels of GAS5 increase (Fig. 1).

The human genome integrity is constantly damaged by genotoxic agents from the exogenous environment (chemicals and ultraviolet, for example) and endogenous environment (replication and oxidative stress) $(51,52)$. The ability to repair damaged DNA is critical for living organisms. GAS5-derived snoRNA expression was induced by DNA damage in a p53-dependent manner in colorectal cancer cell lines (43). It was considered that GAS5-derived snoRNA might be involved in the DNA damage repair.

\section{Implications in cancer management}

Given that aberrant GAS5 expression was reported in various human cancers, it provides an attractive approach for cancer management. Firstly, GAS5 may be used as a biomarker for cancer screening. By monitoring the GAS5 level in an individual with a suspected tumor, it is possible to predict the risk of cancer development and progression, or the prognosis of the cancer. Secondly, GAS5 is assumed to be a tumor suppressor, which inhibits cell growth and metastasis and induces apoptosis in certain cancer cells; therefore, re-introduction of the GAS5 gene provides us with an attractive strategy to treat cancer. 


\section{Conclusions and future directions}

GAS5, downregulated in a number of human cancers, is a well-characterized tumor suppressor. GAS5 induces apoptosis and inhibits proliferation and metastasis. The molecular mechanism of GAS5 tumorigenesis remains to be investigated. Through regulating the expression of various tumor-related genes and proteins, including E2F1 and cyclin D1, GAS5 may exert its function in tumorigenesis. Since the regulatory mechanisms are tissue-specific, the effects of the polymorphisms on GAS5 expression and the associated regulatory mechanisms have only been evaluated in a few cancer tissues. Although previous studies have reported the tumor-suppressing role of GAS5 in several cancer types, including breast and gastric cancer, the expression and function of GAS5 in other tumors, such as liver cancer, remains to be investigated.

\section{References}

1. Li Z, Lei H, Luo M, et al: DNA methylation downregulated mir-10b acts as a tumor suppressor in gastric cancer. Gastric Cancer 18: 43-54, 2014.

2. Oliveira AM, Ross JS and Fletcher JA: Tumor suppressor genes in breast cancer: the gatekeepers and the caretakers. Am J Clin Pathol 124 (Suppl): S16-S28, 2005.

3. Banerjee R, Mani RS, Russo N, et al: The tumor suppressor gene rap1GAP is silenced by miR-101-mediated EZH2 overexpression in invasive squamous cell carcinoma. Oncogene 30: 4339-4349, 2011.

4. Chen X, Cao X, Dong W, Luo S, Suo Z and Jin Y: Expression of TIP30 tumor suppressor gene is down-regulated in human colorectal carcinoma. Dig Dis Sci 55: 2219-2226, 2010.

5. Brosnan CA and Voinnet O: The long and the short of noncoding RNAs. Curr Opin Cell Biol 21: 416-425, 2009.

6. Tano $\mathrm{K}$ and Akimitsu N: Long non-coding RNAs in cancer progression. Front Genet 3: 219, 2012.

7. Kitagawa M, Kotake $\mathrm{Y}$ and Ohhata T: Long non-coding RNAs involved in cancer development and cell fate determination. Curr Drug Targets 13: 1616-1621, 2012.

8. Shi X, Sun M, Liu H, Yao Y and Song Y: Long non-coding RNAs: a new frontier in the study of human diseases. Cancer Lett 339: 159-166, 2013

9. He Y, Meng XM, Huang C, et al: Long noncoding RNAs: Novel insights into hepatocelluar carcinoma. Cancer Lett 344: 20-27, 2014.

10. $\mathrm{Li} \mathrm{CH}$ and Chen Y: Targeting long non-coding RNAs in cancers: progress and prospects. Int J Biochem Cell Biol 45: 1895-1910, 2013.

11. Gutschner $\mathrm{T}$ and Diederichs $\mathrm{S}$ : The hallmarks of cancer: a long non-coding RNA point of view. RNA Biol 9: 703-719, 2012.

12. Smith CM and Steitz JA: Classification of gas5 as a multi-small-nucleolar-RNA (snoRNA) host gene and a member of the 5'-terminal oligopyrimidine gene family reveals common features of snoRNA host genes. Mol Cell Biol 18: 6897-6909, 1998.

13. Muller AJ, Chatterjee S, Teresky A and Levine AJ: The gas5 gene is disrupted by a frameshift mutation within its longest open reading frame in several inbred mouse strains and maps to murine chromosome 1. Mamm Genome 9: 773-774, 1998.

14. Coccia EM, Cicala C, Charlesworth A, et al: Regulation and expression of a growth arrest-specific gene (gas5) during growth, differentiation and development. Mol Cell Biol 12: 3514-3521, 1992.

15. Mourtada-Maarabouni M, Pickard MR, Hedge VL, Farzaneh F and Williams GT: GAS5, a non-protein-coding RNA, controls apoptosis and is downregulated in breast cancer. Oncogene 28: 195-208, 2009.

16. Sun M, Jin FY, Xia R, et al: Decreased expression of long noncoding RNA GAS5 indicates a poor prognosis and promotes cell proliferation in gastric cancer. BMC Cancer 14: 319, 2014.

17. Pickard MR, Mourtada-Maarabouni M and Williams GT: Long non-coding RNA GAS5 regulates apoptosis in prostate cancer cell lines. Biochim Biophys Acta 1832: 1613-1623, 2013.
18. Fleming JV, Fontanier N, Harries DN and Rees WD: The growth arrest genes gas5, gas6 and CHOP-10 (gadd153) are expressed in the mouse preimplantation embryo. Mol Reprod Dev 48: 310-316, 1997.

19. Nakamura Y, Takahashi N, Kakegawa E, et al: The GAS5 (growth arrest-specific transcript 5) gene fuses to BCL6 as a result of $t$ $(1 ; 3)(\mathrm{q} 25 ; \mathrm{q} 27)$ in a patient with B-cell lymphoma. Cancer Genet Cytogenet 182: 144-149, 2008.

20. Tcherkezian J, Cargnello M, Romeo Y, et al: Proteomic analysis of cap-dependent translation identifies LARP1 as a key regulator of 5'TOP mRNA translation. Genes Dev 28: 357-371, 2014.

21. Raho G, Barone V, Rossi D, Philipson L and Sorrentino V: The gas5 gene shows four alternative splicing patterns without coding for a protein. Gene 256: 13-17, 2000.

22. Renganathan A, Kresoja-Rakic J, Echeverry N, et al: GAS5 long non-coding RNA in malignant pleural mesothelioma. Mol Cancer 13: 119, 2014.

23. Shi X, Sun M, Liu H, et al: A critical role for the long non-coding RNA GAS5 in proliferation and apoptosis in non-small-cell lung cancer. Mol Carcinog: Dec 19, 2013 (Epub ahead of print).

24. Mourtada-Maarabouni M, Hasan AM, Farzaneh F and Williams GT: Inhibition of human T-cell proliferation by mammalian target of rapamycin (mTOR) antagonists requires noncoding RNA growth-arrest-specific transcript 5 (GAS5). Mol Pharmacol 78: 19-28, 2010.

25. Mourtada-Maarabouni M, Hedge VL, Kirkham L, Farzaneh F and Williams GT: Growth arrest in human T-cells is controlled by the non-coding RNA growth-arrest-specific transcript 5 (GAS5). J Cell Sci 121: 939-946, 2008.

26. Kino T, Hurt DE, Ichijo T, Nader N and Chrousos GP: Noncoding RNA gas 5 is a growth arrest- and starvation-associated repressor of the glucocorticoid receptor. Sci Signal 3: ra8, 2010.

27. Liu Z, Wang W, Jiang J, et al: Downregulation of GAS5 promotes bladder cancer cell proliferation, partly by regulating CDK6. PLoS One 8: e73991, 2013.

28. Lu X, Fang Y, Wang Z, et al: Downregulation of gas5 increases pancreatic cancer cell proliferation by regulating CDK6. Cell Tissue Res 354: 891-896, 2013.

29. Gee HE, Buffa FM, Camps C, et al: The small-nucleolar RNAs commonly used for microRNA normalisation correlate with tumour pathology and prognosis. Br J Cancer 104: 1168-1177, 2011

30. Zhang Z, Zhu Z, Watabe K, et al: Negative regulation of lncRNA GAS5 by miR-21. Cell Death Differ 20: 1558-1568, 2013.

31. Qiao HP, Gao WS, Huo JX and Yang ZS: Long non-coding RNA GAS5 functions as a tumor suppressor in renal cell carcinoma. Asian Pac J Cancer Prev 14: 1077-1082, 2013.

32. Zhou S, Wang J and Zhang Z: An emerging understanding of long noncoding RNAs in kidney cancer. J Cancer Res Clin Oncol 140: 1989-1995, 2014

33. Shain SA: Exogenous fibroblast growth factors maintain viability, promote proliferation and suppress GADD45alpha and GAS6 transcript content of prostate cancer cells genetically modified to lack endogenous FGF-2. Mol Cancer Res 2: 653-661, 2004.

34. Romanuik TL, Wang G, Morozova O, Delaney A, Marra MA and Sadar MD: LNCaP Atlas: gene expression associated with in vivo progression to castration-recurrent prostate cancer. BMC Med Genomics 3: 43, 2010.

35. Abbah SA, Lam CX, Ramruttun AK, Goh JC and Wong HK: Fusion performance of low-dose recombinant human bone morphogenetic protein 2 and bone marrow-derived multipotent stromal cells in biodegradable scaffolds: a comparative study in a large animal model of anterior lumbar interbody fusion. Spine (Phila Pa 1976) 36: 1752-1759, 2011.

36. Agirre X, Vilas-Zornoza A, Jiménez-Velasco A, et al: Epigenetic silencing of the tumor suppressor microRNA Hsa-miR-124a regulates CDK6 expression and confers a poor prognosis in acute lymphoblastic leukemia. Cancer Res 69: 4443-4453, 2009.

37. Mourtada-Maarabouni $M$ and Williams GT: Role of GAS5 noncoding RNA in mediating the effects of rapamycin and its analogues on mantle cell lymphoma cells. Clin Lymphoma Myeloma Leuk 14: 468-473, 2014.

38. Wu RS, Wu KC, Yang JS, et al: Etomidate induces cytotoxic effects and gene expression in a murine leukemia macrophage cell line (RAW264.7). Anticancer Res 31: 2203-2208, 2011.

39. He JF, Luo YM, Wan XH and Jiang D: Biogenesis of MiRNA-195 and its role in biogenesis, the cell cycle and apoptosis. J Biochem Mol Toxicol 25: 404-408, 2011.

40. Pickard MR and Williams GT: Regulation of apoptosis by long non-coding RNA GAS5 in breast cancer cells: implications for chemotherapy. Breast Cancer Res Treat 145: 359-370, 2014. 
41. Zhang J, Zhang P, Wang L, Piao HL and Ma L: Long non-coding RNA HOTAIR in carcinogenesis and metastasis. Acta Biochim Biophys Sin (Shanghai) 46: 1-5, 2014.

42. Li Z, Shen J, Wu WK, et al: Leptin induces cyclin D1 expression and proliferation of human nucleus pulposus cells via JAK/STAT, PI3K/Akt and MEK/ERK pathways. PloS One 7: e53176, 2012.

43. Krell J, Frampton AE, Mirnezami R, et al: Growth arrest-specific transcript 5 associated snoRNA levels are related to p53 expression and DNA damage in colorectal cancer. PloS One 9: e98561, 2014.

44. Okada N, Lin CP, Ribeiro MC, et al: A positive feedback between p53 and miR-34 miRNAs mediates tumor suppression. Genes Dev 28: 438-450, 2014.

45. Choi OR and Lim IK: Loss of p21 (Sdi1) expression in senescent cells after DNA damage accompanied with increase of miR-93 expression and reduced p53 interaction with p21 (Sdi1) gene promoter. Biochem Biophys Res Commun 407: 406-411, 2011.

46. Baldus SE, Schneider PM, Mönig SP, et al: p21/waf1/cip1 in gastric cancer: associations with histopathological subtypes, lymphonodal metastasis, prognosis and p53 status. Scand J Gastroenterol 36: 975-980, 2001.
47. Wu J, Qian J, Li C, et al: miR-129 regulates cell proliferation by downregulating Cdk6 expression. Cell Cycle 9: 1809-1818, 2010.

48. Rodriguez-Otero P, Román-Gómez J, Vilas-Zornoza A, et al: Deregulation of FGFR1 and CDK6 oncogenic pathways in acute lymphoblastic leukaemia harbouring epigenetic modifications of the MIR9 family. Br J Haematol 155: 73-83, 2011.

49. Dadras SS, Skrzypek A, Nguyen L, et al: Prox-1 promotes invasion of kaposiform hemangioendotheliomas. J Invest Dermatol 128: 2798-2806, 2008.

50. Wu I, Shin SC, Cao Y, et al: Selective glucocorticoid receptor translational isoforms reveal glucocorticoid-induced apoptotic transcriptomes. Cell Death Dis 4: e453, 2013.

51. Ghosal G and Chen J: DNA damage tolerance: a double-edged sword guarding the genome. Transl Cancer Res 2: 107-129, 2013.

52. Alexeyev M, Shokolenko I, Wilson G and LeDoux S: The maintenance of mitochondrial DNA integrity-critical analysis and update. Cold Spring Harb Perspect Biol 5: a012641, 2013. 